\title{
Structural Biology in the Abyss: SEC-SAXS at Deep Ocean Pressures
}

\author{
Richard E Gillilan ${ }^{\mathrm{a}}$ \\ aCornell High Energy Synchrotron Source, 161 Synchrotron Drive, Ithaca, NY 14853, \\ USA, reg8@cornell.edu
}

Recent decades of scientific discovery have taught us that life on Earth is far richer and more resilient than we ever imaged. Microbial communities exist and even thrive in some of the most extreme environments yet explored: the deepest mine shafts and wells, corrosive hot springs, deep hydrothermal vents spewing superheated water, permafrost at $-17 \mathrm{C}$, ocean trenches $11 \mathrm{~km}$ deep, and even coal beds and porous rock multiple kilometers below the sea floor. Given the most recent surveys of global biomass, most life on Earth may, in fact, live under high pressure. Phylogenetic evidence even suggests that our first single-celled ancestors may have developed in hydrothermal vents in the deep ancient ocean. If this is the case, much of our foundational molecular biology may need to be re-examined in the context of our high-pressure origins. While high-pressure biology has been a subject of study for many years, precious little high-pressure molecular structural information has yet been obtained. This talk will introduce the new CHESS-U high-pressure biology facility (HP-Bio) dedicated to providing the growing deep-life community with easily-accessible HP-SAXS and HP-MX technology. While the innovations in flow cells and size-exclusion chromatography coupled SAXS (SEC-SAXS) have revolutionized conventional BioSAXS data collection, current high-pressure SAXS cell designs still rely upon static non-flowing samples that are vulnerable to radiation damage. In this talk, I will show that the highly-popular and powerful SECSAXS technique can be performed at deep ocean pressures (100 MPa) as easily and rapidly as at normal ambient pressures. Though observable changes in bed packing can occur, the fundamental mechanism of size-exclusion, at least for silica-based stationary phases, appears to be preserved at these pressures. The advantages of SEC-SAXS are therefore fully available for the study of biomolecular systems at all ocean depths. I will discuss the unique features of high-pressure SAXS data, the potential for extending the method to other types of chromatography, and the potential for reaching even higher pressures where de-aggregation and unfolding phenomena are more pronounced. 